\title{
ON A TWO-POINT BOUNDARY VALUE PROBLEM FOR THIRD-ORDER LINEAR FUNCTIONAL DIFFERENTIAL EQUATIONS. PART II.
}

\author{
ROBERT HAKL
}

\begin{abstract}
Efficient conditions sufficient for the solvability of the problem $u^{\prime \prime \prime}(t)=g(t) u(\mu(t))-p(t) u(\tau(t))+q(t) ; \quad u(a)=c_{1}, \quad u^{\prime}(a)=c_{2}, \quad u(b)=c_{3}$ are derived using the general results obtained in our recent paper [1]. Here, $p, g \in$ $L\left([a, b] ; \mathbb{R}^{+}\right), q \in L([a, b] ; \mathbb{R}), \tau, \mu:[a, b] \rightarrow[a, b]$ are measurable functions, and $c_{i} \in \mathbb{R}(i=1,2,3)$. Sign-constant solutions are discussed as well.
\end{abstract}

\section{INTRODUCTION}

In [1], we have obtained general results on the existence, uniqueness and positivity of a solution to the two-point boundary value problem

$$
\begin{aligned}
& u^{\prime \prime \prime}(t)=\ell(u)(t)+q(t) \quad \text { for a.e. } t \in[a, b], \\
& u(a)=c_{1}, \quad u^{\prime}(a)=c_{2}, \quad u(b)=c_{3},
\end{aligned}
$$

where $q \in L([a, b] ; \mathbb{R}), c_{i} \in \mathbb{R}(i=1,2,3)$, and $\ell: C([a, b] ; \mathbb{R}) \rightarrow L([a, b] ; \mathbb{R})$ is a linear bounded operator. The present paper, which is the second part of [1], contains some nontrivial consequences of the general results of [1] for the equations with deviating arguments. The proofs essentially use the statements obtained in [1]. We refer to [1] for an overview of the topic and the related literature.

Here, we consider the problem (1.1), (1.2) with the operator $\ell$ having one of the following forms:

$$
\begin{aligned}
& \ell(v)(t)=-p(t) v(\tau(t)) \quad \text { for a.e. } t \in[a, b], \quad v \in C([a, b] ; \mathbb{R}), \\
& \ell(v)(t)=g(t) v(\mu(t)) \quad \text { for a.e. } t \in[a, b], \quad v \in C([a, b] ; \mathbb{R}),
\end{aligned}
$$

and

$$
\ell(v)(t)=g(t) v(\mu(t))-p(t) v(\tau(t)) \quad \text { for a.e. } t \in[a, b], \quad v \in C([a, b] ; \mathbb{R}),
$$

where $p, g \in L\left([a, b] ; \mathbb{R}^{+}\right)$and $\tau, \mu:[a, b] \rightarrow[a, b]$ are measurable functions. By a solution to the problem (1.1), (1.2), we understand a function $u:[a, b] \rightarrow \mathbb{R}$ which is absolutely continuous together with its first and second derivatives, satisfies the equality (1.1) almost everywhere in $[a, b]$, and (1.2) holds.

$M S C$ (2010): primary 34K06, 34K10.

Keywords: linear functional differential equation, boundary value problem, positive solution.

The research was supported by RVO: 67985840 . 
The following notation is used throughout the paper:

$\mathbb{R}$ is a set of all real numbers, $\mathbb{R}^{+}=[0,+\infty[$.

$C([a, b] ; \mathbb{R})$ is a Banach space of all continuous functions $u:[a, b] \rightarrow \mathbb{R}$ endowed with the norm

$$
\|u\|_{C}=\max \{|u(t)|: t \in[a, b]\} .
$$

$\widetilde{C}^{2}([a, b] ; \mathbb{R})$ is the set of all functions $u:[a, b] \rightarrow \mathbb{R}$ that are absolutely continuous together with their first and second derivatives.

$\widetilde{C}_{l o c}^{2}(] a, b[; \mathbb{R})$ is the set of all functions $\left.u:\right] a, b\left[\rightarrow \mathbb{R}\right.$ such that $u \in \widetilde{C}^{2}([\alpha, \beta] ; \mathbb{R})$ for every $\alpha, \beta \in] a, b[, \alpha<\beta$.

Let $u:] a, b[\rightarrow \mathbb{R}$ be a continuous function and let there exist a finite or an infinite right, left, limit of $u$ at the point $a, b$, respectively. Then we will write $u(a+), u(b-)$, instead of $\lim _{t \rightarrow a+} u(t), \lim _{t \rightarrow b-} u(t)$, respectively.

$\widetilde{C}_{0}(] a, b[; \mathbb{R})$ is a set of all functions $u \in \widetilde{C}_{l o c}^{2}(] a, b[; \mathbb{R}) \cap C([a, b] ; \mathbb{R})$ such that there exist finite or infinite limits $u^{\prime}(a+)$ and $u^{\prime}(b-)$.

$L([a, b] ; \mathbb{R})$ is a Banach space of all Lebesgue integrable functions $p:[a, b] \rightarrow \mathbb{R}$ endowed with the norm

$$
\|p\|_{L}=\int_{a}^{b}|p(s)| \mathrm{d} s .
$$

$L\left([a, b] ; \mathbb{R}^{+}\right)=\left\{p \in L([a, b] ; \mathbb{R}): p(t) \in \mathbb{R}^{+}\right.$for a.e. $\left.t \in[a, b]\right\}$.

$\mathcal{L}_{a b}$ is a set of all linear bounded operators $\ell: C([a, b] ; \mathbb{R}) \rightarrow L([a, b] ; \mathbb{R})$.

For convenience we recall the definitions introduced in [1].

Definition 1.1. An operator $\ell \in \mathcal{L}_{a b}$ is said to belong to the set $\mathcal{V}([a, b])$ if every function $u \in \widetilde{C}^{2}([a, b] ; \mathbb{R})$ satisfying

$$
\begin{aligned}
& u^{\prime \prime \prime}(t) \leq \ell(u)(t) \quad \text { for a.e. } t \in[a, b] \\
& u(a) \geq 0, \quad u^{\prime}(a) \geq 0, \quad u(b) \geq 0
\end{aligned}
$$

admits the inequality

$$
u(t) \geq 0 \quad \text { for } t \in[a, b] .
$$

Definition 1.2. An operator $\ell \in \mathcal{L}_{a b}$ is said to belong to the set $\mathcal{V}_{0}([a, b])$ if every function $u \in \widetilde{C}^{2}([a, b] ; \mathbb{R})$ satisfying (1.6) and

$$
u(a)=0, \quad u^{\prime}(a) \geq 0, \quad u(b)=0
$$

admits the inequality (1.7). 


\section{MAin Results}

Theorem 2.1. Let

$$
\begin{aligned}
& (b-\tau(t))(\tau(t)-a) \int_{a}^{\tau(t)}(b-s)(s-a) p(s) \mathrm{d} s-\frac{(b-\tau(t))^{2}}{2} \int_{a}^{\tau(t)}(s-a)^{2} p(s) \mathrm{d} s \\
& +\frac{(\tau(t)-a)^{2}}{2} \int_{\tau(t)}^{b}(b-s)^{2} p(s) \mathrm{d} s<(b-a)^{2} \quad \text { for a.e. } t \in[a, b] .
\end{aligned}
$$

Then the operator $\ell$ defined by (1.3) belongs to the set $\mathcal{V}([a, b])$.

Corollary 2.2. Let

$$
\int_{a}^{b} p(s) \mathrm{d} s \leq \frac{16}{(b-a)^{2}}
$$

Then the operator $\ell$ defined by (1.3) belongs to the set $\mathcal{V}([a, b])$.

Theorem 2.3. Let

$$
\left(\frac{b-\tau(t)}{b-t}\right)^{1-\frac{\sqrt{3}}{3}}\left(\frac{\tau(t)-a}{t-a}\right)^{1+\frac{\sqrt{3}}{3}} p(t) \leq \frac{2 \sqrt{3}(b-a)^{3}}{9(b-t)^{3}(t-a)^{3}}
$$

Then the operator $\ell$ defined by (1.3) belongs to the set $\mathcal{V}([a, b])$.

Theorem 2.4. Let there exist $c \in[a, b]$ and $\lambda_{i j} \in \mathbb{R}^{+}, \nu_{i} \in[0,1[,(i, j=1,2)$ such that

$$
\begin{aligned}
& \int_{0}^{+\infty} \frac{\mathrm{d} s}{s^{2}+\lambda_{11} s+\lambda_{12}} \geq \frac{(c-a)^{1-\nu_{1}}}{1-\nu_{1}}, \\
& \int_{0}^{+\infty} \frac{\mathrm{d} s}{s^{2}+\lambda_{21} s+\lambda_{22}} \geq \frac{(b-c)^{1-\nu_{2}}}{1-\nu_{2}},
\end{aligned}
$$

and

$$
\begin{aligned}
& -p(t) \frac{(t-a)^{2}}{2}+p(t) \sigma(t) \frac{(\tau(t)-t)^{2}}{2} \leq \frac{\nu_{1}}{t-a}+\frac{\lambda_{11}}{(t-a)^{\nu_{1}}} \quad \text { for a.e. } t \in[a, c] \\
& p(t)(t-a)+p(t) \sigma(t)(\tau(t)-t) \leq \frac{\lambda_{12}}{(t-a)^{2 \nu_{1}}} \quad \text { for a.e. } t \in[a, c] \\
& p(t) \frac{(t-a)^{2}}{2}-p(t) \sigma(t) \frac{(\tau(t)-t)^{2}}{2} \leq \frac{\nu_{2}}{b-t}+\frac{\lambda_{21}}{(b-t)^{\nu_{2}}} \quad \text { for a.e. } t \in[c, b] \\
& p(t)(t-a)+p(t) \sigma(t)(\tau(t)-t) \leq \frac{\lambda_{22}}{(b-t)^{2 \nu_{2}}} \quad \text { for a.e. } t \in[c, b]
\end{aligned}
$$

where

$$
\sigma(t)=\frac{1}{2}(1+\operatorname{sgn}(\tau(t)-t))
$$


Then the operator $\ell$ defined by (1.3) belongs to the set $\mathcal{V}([a, b])$.

Theorem 2.5. Let $\mu(t) \leq t$ for a.e. $t \in[a, b]$ and

$$
\int_{a}^{b}(b-s)(s-a) g(s) \mathrm{d} s \leq 2 .
$$

Then the operator $\ell$ defined by (1.4) belongs to the set $\mathcal{V}([a, b])$.

Corollary 2.6. Let $\mu(t) \leq t$ for a.e. $t \in[a, b]$ and

$$
\int_{a}^{b} g(s) \mathrm{d} s \leq \frac{8}{(b-a)^{2}} .
$$

Then the operator $\ell$ defined by (1.4) belongs to the set $\mathcal{V}([a, b])$.

Theorem 2.7. Let $\mu(t) \leq t$ for a.e. $t \in[a, b]$ and

$$
\begin{array}{r}
\left(\frac{b-\mu(t)}{b-t}\right)^{1+\frac{\sqrt{3}}{3}}\left(\frac{\mu(t)-a+\omega}{t-a+\omega}\right)^{1-\frac{\sqrt{3}}{3}} g(t) \leq \frac{2 \sqrt{3}(b-a+\omega)^{3}}{9(b-t)^{3}(t-a+\omega)^{3}} \\
\text { for a.e. } t \in[a, b]
\end{array}
$$

with $\omega=\frac{3-\sqrt{3}}{3+\sqrt{3}}(b-a)$. Then the operator $\ell$ defined by (1.4) belongs to the set $\mathcal{V}([a, b])$.

Theorem 2.8. Let $\mu(t) \leq t$ for a.e. $t \in[a, b]$. Let, moreover, there exist $c \in[a, b]$ and $\lambda_{i j} \in \mathbb{R}^{+}, \nu_{i} \in[0,1[,(i, j=1,2)$ such that (2.4), (2.5) hold and

$$
\begin{aligned}
& g(t) \frac{(b-t)^{2}}{2}-g(t) \frac{(t-\mu(t))^{2}}{2} \leq \frac{\nu_{1}}{t-a}+\frac{\lambda_{11}}{(t-a)^{\nu_{1}}} \quad \text { for a.e. } t \in[a, c], \\
& g(t)(b-t)+g(t)(t-\mu(t)) \leq \frac{\lambda_{12}}{(t-a)^{2 \nu_{1}}} \quad \text { for a.e. } t \in[a, c], \\
- & g(t) \frac{(b-t)^{2}}{2}+g(t) \frac{(t-\mu(t))^{2}}{2} \leq \frac{\nu_{2}}{b-t}+\frac{\lambda_{21}}{(b-t)^{\nu_{2}}} \quad \text { for a.e. } t \in[c, b], \\
& g(t)(b-t)+g(t)(t-\mu(t)) \leq \frac{\lambda_{22}}{(b-t)^{2 \nu_{2}}} \quad \text { for a.e. } t \in[c, b] .
\end{aligned}
$$

Then the operator $\ell$ defined by (1.4) belongs to the set $\mathcal{V}([a, b])$.

Theorem 2.9. Let $\mu(t) \leq t$ for a.e. $t \in[a, b]$ and

$$
\begin{gathered}
(b-\mu(t))(\mu(t)-a) \int_{\mu(t)}^{b}(b-s)(s-a) g(s) \mathrm{d} s-\frac{(\mu(t)-a)^{2}}{2} \int_{\mu(t)}^{b}(b-s)^{2} g(s) \mathrm{d} s \\
+\frac{(b-\mu(t))^{2}}{2} \int_{a}^{\mu(t)}(s-a)^{2} g(s) \mathrm{d} s \leq(b-a)^{2} \quad \text { for a.e. } t \in[a, b] .
\end{gathered}
$$

Then the operator $\ell$ defined by (1.4) belongs to the set $\mathcal{V}_{0}([a, b])$. 
Corollary 2.10. Let $\mu(t) \leq t$ for a.e. $t \in[a, b]$ and

$$
\int_{a}^{b} g(s) \mathrm{d} s \leq \frac{16}{(b-a)^{2}} .
$$

Then the operator $\ell$ defined by (1.4) belongs to the set $\mathcal{V}_{0}([a, b])$.

Theorem 2.11. Let $\mu(t) \leq t$ for a.e. $t \in[a, b]$ and

$$
\begin{aligned}
\left(\frac{b-\mu(t)}{b-t}\right)^{1+\frac{\sqrt{3}}{3}}\left(\frac{\mu(t)-a}{t-a}\right)^{1-\frac{\sqrt{3}}{3}} g(t) \leq \frac{2 \sqrt{3}(b-a)^{3}}{9(b-t)^{3}(t-a)^{3}} \\
\text { for a.e. } t \in[a, b] .
\end{aligned}
$$

Then the operator $\ell$ defined by (1.4) belongs to the set $\mathcal{V}_{0}([a, b])$.

The results listed below immediately follow from [1, Theorems 2.10-2.13], Theorems 2.1-2.11, and Corollaries 2.2-2.10.

Theorem 2.12. Let functions $p, \tau$ satisfy the assumptions of at least one of Theorems 2.1-2.4 or Corollary 2.2 and let functions $g, \mu$ satisfy the assumptions of at least one of Theorems 2.5-2.8 or Corollary 2.6. Then the problem (1.1), (1.2) with $\ell$ defined by (1.5) has a unique solution $u$. If, in addition,

$$
\begin{aligned}
& q(t) \leq 0 \quad \text { for a.e. } t \in[a, b], \\
& c_{i} \geq 0 \quad(i=1,2,3), \\
& \|q\|_{L}+\sum_{i=1}^{3} c_{i}>0
\end{aligned}
$$

then

$$
u(t)>0 \quad \text { for } t \in] a, b[.
$$

Theorem 2.13. Let functions $p, \tau$ satisfy the assumptions of at least one of Theorems 2.1-2.4 or Corollary 2.2 and let functions $g, \mu$ satisfy the assumptions of either Theorem 2.9 or Theorem 2.11 or Corollary 2.10. Then the problem (1.1), (1.2) with $\ell$ defined by (1.5) has a unique solution $u$. If, in addition, (2.19) holds and

$$
\begin{aligned}
& c_{1}=0, \quad c_{2} \geq 0, \quad c_{3}=0, \\
& \|q\|_{L}+c_{2}>0
\end{aligned}
$$

then (2.20) holds.

Theorem 2.14. Let functions $p, \tau$ satisfy the assumptions of at least one of Theorems 2.1-2.4 or Corollary 2.2 and let functions $g, \mu$ satisfy at least one of the following items:

(i) $\mu(t) \leq t$ for a.e. $t \in[a, b]$ and

$$
\int_{a}^{b} g(s) \mathrm{d} s \leq \frac{32}{(b-a)^{2}}
$$


(ii) $\mu(t) \leq t$ for a.e. $t \in[a, b]$ and

$$
\begin{aligned}
& (b-\mu(t))(\mu(t)-a) \int_{\mu(t)}^{b}(b-s)(s-a) g(s) \mathrm{d} s-\frac{(\mu(t)-a)^{2}}{2} \int_{\mu(t)}^{b}(b-s)^{2} g(s) \mathrm{d} s \\
& +\frac{(b-\mu(t))^{2}}{2} \int_{a}^{\mu(t)}(s-a)^{2} g(s) \mathrm{d} s \leq 2(b-a)^{2} \quad \text { for a.e. } t \in[a, b] ;
\end{aligned}
$$

(iii) $\mu(t) \leq t$ for a.e. $t \in[a, b]$ and

$$
\left(\frac{b-\mu(t)}{b-t}\right)^{1+\frac{\sqrt{3}}{3}}\left(\frac{\mu(t)-a}{t-a}\right)^{1-\frac{\sqrt{3}}{3}} g(t) \leq \frac{4 \sqrt{3}(b-a)^{3}}{9(b-t)^{3}(t-a)^{3}} \quad \text { for a.e. } t \in[a, b]
$$

(iv) $\mu(t) \leq t$ for a.e. $t \in[a, b]$ and there exist $c \in[a, b]$ and $\lambda_{i j} \in \mathbb{R}^{+}$, $\nu_{i} \in[0,1[,(i, j=1,2)$ such that $(2.4),(2.5)$ hold and

$$
\begin{gathered}
g(t) \frac{(b-t)^{2}}{2}-g(t) \frac{(t-\mu(t))^{2}}{2} \leq \frac{2 \nu_{1}}{t-a}+\frac{2 \lambda_{11}}{(t-a)^{\nu_{1}}} \quad \text { for a.e. } t \in[a, c], \\
g(t)(b-t)+g(t)(t-\mu(t)) \leq \frac{2 \lambda_{12}}{(t-a)^{2 \nu_{1}}} \quad \text { for a.e. } t \in[a, c], \\
-g(t) \frac{(b-t)^{2}}{2}+g(t) \frac{(t-\mu(t))^{2}}{2} \leq \frac{2 \nu_{2}}{b-t}+\frac{2 \lambda_{21}}{(b-t)^{\nu_{2}}} \quad \text { for a.e. } t \in[c, b], \\
g(t)(b-t)+g(t)(t-\mu(t)) \leq \frac{2 \lambda_{22}}{(b-t)^{2 \nu_{2}}} \quad \text { for a.e. } t \in[c, b] .
\end{gathered}
$$

Then the problem (1.1), (1.2) with $\ell$ defined by (1.5) is uniquely solvable.

Theorem 2.15. Let functions $p, \tau$ satisfy the assumptions of at least one of Theorems 2.1-2.4 or Corollary 2.2. Let, moreover, $\tau(t) \leq t$ and $\mu(t) \leq t$ for a.e. $t \in[a, b]$. Then the problem (1.1), (1.2) with $\ell$ defined by (1.5) is uniquely solvable.

\section{Proofs}

Proof of Theorem 2.1. If $p \equiv 0$, then the conclusion of theorem follows from [1, Remark 2.3]. Therefore, we can assume that

$$
\int_{a}^{b} p(s) \mathrm{d} s>0
$$

Put

$$
\begin{aligned}
& \gamma(t)=\frac{1}{(b-a)^{2}}\left((b-t)(t-a) \int_{a}^{t}(b-s)(s-a) p(s) \mathrm{d} s\right. \\
& \left.-\frac{(b-t)^{2}}{2} \int_{a}^{t}(s-a)^{2} p(s) \mathrm{d} s+\frac{(t-a)^{2}}{2} \int_{t}^{b}(b-s)^{2} p(s) \mathrm{d} s\right) \quad \text { for } t \in[a, b] .
\end{aligned}
$$


We will show that $\gamma$ satisfies the assumptions of [1, Theorem 2.1] with $\ell$ defined by (1.3). It can be easily verified that

$$
\begin{aligned}
& \gamma^{\prime \prime \prime}(t)=-p(t) \quad \text { for a.e. } t \in[a, b], \\
& \gamma(a)=0, \quad \gamma^{\prime}(a)=0, \quad \gamma(b)=0 .
\end{aligned}
$$

Therefore, according to [1, Remark 2.3, Theorem 2.10] and the inequality (3.1), we have

$$
\gamma(t)>0 \quad \text { for } t \in] a, b[\text {. }
$$

Furthermore, (2.1) and (3.2) imply

$$
\gamma(\tau(t))<1 \quad \text { for a.e. } t \in[a, b],
$$

which, when used in (3.3), yields

$$
\begin{aligned}
& \gamma^{\prime \prime \prime}(t) \leq-p(t) \gamma(\tau(t)) \quad \text { for a.e. } t \in[a, b] \\
& \text { meas }\left\{t \in[a, b]: \gamma^{\prime \prime \prime}(t)<-p(t) \gamma(\tau(t))\right\}>0
\end{aligned}
$$

Finally, $\gamma \in \widetilde{C}_{0}(] a, b[; \mathbb{R})$ and $(3.4)$, (3.5), (3.7), and (3.8) imply that all the assumptions of $[1$, Theorem 2.1] are fulfilled.

Proof of Corollary 2.2. If $p \equiv 0$, then the conclusion of the corollary follows from [1, Remark 2.3]. Therefore, assume that (3.1) holds. It is sufficient to show that (2.1) is fulfilled. For this purpose, we will estimate the maximum value of the function $\gamma$ defined by (3.2). Obviously, (3.3)-(3.5) hold. In view of (3.4) and (3.5), there exists $\left.t_{0} \in\right] a, b[$ such that

$$
\gamma\left(t_{0}\right)=\max \{\gamma(t): t \in[a, b]\} .
$$

Consequently, $\gamma^{\prime}\left(t_{0}\right)=0$, i.e.,

$$
\begin{aligned}
& \left(a+b-2 t_{0}\right) \int_{a}^{t_{0}}(b-s)(s-a) p(s) \mathrm{d} s+\left(b-t_{0}\right) \int_{a}^{t_{0}}(s-a)^{2} p(s) \mathrm{d} s \\
& +\left(t_{0}-a\right) \int_{t_{0}}^{b}(b-s)^{2} p(s) \mathrm{d} s=0 .
\end{aligned}
$$

From (3.10) we obtain

$$
\begin{aligned}
& \left(t_{0}-a\right) \int_{a}^{t_{0}}(b-s)(s-a) p(s) \mathrm{d} s-\left(b-t_{0}\right) \int_{a}^{t_{0}}(s-a)^{2} p(s) \mathrm{d} s \\
= & \left(t_{0}-a\right) \int_{t_{0}}^{b}(b-s)^{2} p(s) \mathrm{d} s+\left(b-t_{0}\right) \int_{a}^{t_{0}}(b-s)(s-a) p(s) \mathrm{d} s .
\end{aligned}
$$


From (3.2) we have

$$
\begin{gathered}
\gamma\left(t_{0}\right)=\frac{t_{0}-a}{2(b-a)^{2}}\left(\left(t_{0}-a\right) \int_{t_{0}}^{b}(b-s)^{2} p(s) \mathrm{d} s+\left(b-t_{0}\right) \int_{a}^{t_{0}}(b-s)(s-a) p(s) \mathrm{d} s\right) \\
+\frac{b-t_{0}}{2(b-a)^{2}}\left(\left(t_{0}-a\right) \int_{a}^{t_{0}}(b-s)(s-a) p(s) \mathrm{d} s-\left(b-t_{0}\right) \int_{a}^{t_{0}}(s-a)^{2} p(s) \mathrm{d} s\right) .
\end{gathered}
$$

Now using (3.11) in the latter equality, we obtain

$$
\gamma\left(t_{0}\right)=\frac{t_{0}-a}{2(b-a)} \int_{t_{0}}^{b}(b-s)^{2} p(s) \mathrm{d} s+\frac{b-t_{0}}{2(b-a)} \int_{a}^{t_{0}}(b-s)(s-a) p(s) \mathrm{d} s,
$$

whence, on account of the relation $4 A B \leq(A+B)^{2}$, we get

$$
\begin{aligned}
& \gamma\left(t_{0}\right) \leq \frac{\left(b-t_{0}\right)^{2}\left(t_{0}-a\right)}{2(b-a)} \int_{t_{0}}^{b} p(s) \mathrm{d} s+\frac{(b-a)\left(b-t_{0}\right)}{8} \int_{a}^{t_{0}} p(s) \mathrm{d} s \\
& \leq \frac{(b-a)\left(b-t_{0}\right)}{8} \int_{a}^{b} p(s) \mathrm{d} s .
\end{aligned}
$$

On the other hand, the equality (3.10) yields

$$
a+b-2 t_{0}<0, \quad \text { i.e. } \quad t_{0}>\frac{a+b}{2} .
$$

Therefore, the inequality (3.12) with respect to (2.2), (3.1), and (3.13) results in

$$
\gamma\left(t_{0}\right)<\frac{(b-a)^{2}}{16} \int_{a}^{b} p(s) \mathrm{d} s \leq 1
$$

Now in view of (3.9), we have (3.6), whence, on account of (3.2), we get (2.1).

Proof of Theorem 2.3. Put

$$
\gamma(t)=(b-t)^{1-\frac{\sqrt{3}}{3}}(t-a)^{1+\frac{\sqrt{3}}{3}} \quad \text { for } t \in[a, b] .
$$

Obviously, $\gamma \in \widetilde{C}_{0}(] a, b[; \mathbb{R}), \gamma(t)>0$ for $\left.t \in\right] a, b[$,

$$
\gamma(a)=0, \quad \gamma^{\prime}(a)=0, \quad \gamma(b)=0,
$$

and

$$
\gamma^{\prime \prime \prime}(t)=-\frac{2 \sqrt{3}(b-a)^{3}}{9(b-t)^{3}(t-a)^{3}}(b-t)^{1-\frac{\sqrt{3}}{3}}(t-a)^{1+\frac{\sqrt{3}}{3}} \quad \text { for a.e. } t \in[a, b] .
$$

Using (2.3) in the latter equality, in view of (3.15), we get

$$
\gamma^{\prime \prime \prime}(t) \leq-p(t) \gamma(\tau(t)) \quad \text { for a.e. } t \in[a, b] .
$$

Moreover, (3.8) holds because $p(\cdot) \gamma(\tau(\cdot)) \in L([a, b] ; \mathbb{R})$ and $\gamma^{\prime \prime \prime} \notin L([a, b] ; \mathbb{R})$. Thus, all the assumptions of [1, Theorem 2.1] are fulfilled. 
Proof of Theorem 2.4. Assume $c \in] a, b[$; the cases $c=a$ and $c=b$ can be proved analogously. Without loss of generality we can assume that (2.4) and (2.5) are fulfilled as equalities. Define functions $\rho_{i}(i=1,2)$ as follows:

$$
\begin{gathered}
\left.\left.\int_{\rho_{1}(t)}^{+\infty} \frac{\mathrm{d} s}{s^{2}+\lambda_{11} s+\lambda_{12}}=\frac{(t-a)^{1-\nu_{1}}}{1-\nu_{1}} \quad \text { for } t \in\right] a, c\right], \\
\int_{\rho_{2}(t)}^{+\infty} \frac{\mathrm{d} s}{s^{2}+\lambda_{21} s+\lambda_{22}}=\frac{(b-t)^{1-\nu_{2}}}{1-\nu_{2}} \quad \text { for } t \in[c, b[.
\end{gathered}
$$

Then

$$
\begin{aligned}
& \left.\rho_{1}(t)>0 \quad \text { for } t \in\right] a, c\left[, \quad \rho_{2}(t)>0 \quad \text { for } t \in\right] c, b[ \\
& \rho_{i}(c)=0 \quad(i=1,2), \quad \lim _{t \rightarrow a+} \rho_{1}(t)=+\infty,
\end{aligned} \quad \lim _{t \rightarrow b-} \rho_{2}(t)=+\infty,
$$

and

$$
\begin{aligned}
& \left.\left.\rho_{1}^{\prime}(t)=-(t-a)^{-\nu_{1}}\left(\rho_{1}^{2}(t)+\lambda_{11} \rho_{1}(t)+\lambda_{12}\right) \quad \text { for } t \in\right] a, c\right] \text {, } \\
& \rho_{2}^{\prime}(t)=(b-t)^{-\nu_{2}}\left(\rho_{2}^{2}(t)+\lambda_{21} \rho_{2}(t)+\lambda_{22}\right) \quad \text { for } t \in[c, b[\text {. }
\end{aligned}
$$

Put

$$
z(t)= \begin{cases}\exp \left(-\int_{t}^{c}(s-a)^{-\nu_{1}} \rho_{1}(s) \mathrm{d} s\right) & \text { for } t \in] a, c] \\ \exp \left(-\int_{c}^{t}(b-s)^{-\nu_{2}} \rho_{2}(s) \mathrm{d} s\right) & \text { for } t \in] c, b[\end{cases}
$$

and

$$
\gamma(t)=\int_{a}^{t} z(s) \mathrm{d} s \quad \text { for } t \in[a, b] .
$$

We will show that $\gamma$ satisfies the assumptions of [1, Theorem 2.1]. Obviously, $\gamma \in \widetilde{C}_{0}(] a, b[; \mathbb{R})$ and

$$
\gamma(a)=0, \quad \gamma(t)>0 \quad \text { for } t \in] a, b] .
$$

Moreover, in view of (3.19) and (3.22), we have

$$
\gamma^{\prime}(a+)=0
$$

Furthermore, (3.22) and (3.23) yield

$$
\gamma^{\prime \prime}(t)= \begin{cases}(t-a)^{-\nu_{1}} \rho_{1}(t) \gamma^{\prime}(t) & \text { for } t \in] a, c] \\ -(b-t)^{-\nu_{2}} \rho_{2}(t) \gamma^{\prime}(t) & \text { for } t \in] c, b[\end{cases}
$$

Obviously,

$$
\left.\gamma^{\prime}(t)>0 \quad \text { for } t \in\right] a, b[
$$

and, in view of (3.18), we have

$$
\left.\gamma^{\prime \prime}(t)>0 \quad \text { for } t \in\right] a, c\left[, \quad \gamma^{\prime \prime}(t)<0 \quad \text { for } t \in\right] c, b[\text {. }
$$


Finally, with respect to (3.20) or (3.21), from (3.26), we obtain

$$
\begin{aligned}
& \gamma^{\prime \prime \prime}(t)=-\nu_{1}(t-a)^{-\nu_{1}-1} \rho_{1}(t) \gamma^{\prime}(t)-(t-a)^{-2 \nu_{1}} \lambda_{11} \rho_{1}(t) \gamma^{\prime}(t) \\
& \left.\left.-(t-a)^{-2 \nu_{1}} \lambda_{12} \gamma^{\prime}(t) \quad \text { for } t \in\right] a, c\right],
\end{aligned}
$$

or

$$
\begin{aligned}
& \gamma^{\prime \prime \prime}(t)=-\nu_{2}(b-t)^{-\nu_{2}-1} \rho_{2}(t) \gamma^{\prime}(t)-(b-t)^{-2 \nu_{2}} \lambda_{21} \rho_{2}(t) \gamma^{\prime}(t) \\
& \left.-(b-t)^{-2 \nu_{2}} \lambda_{22} \gamma^{\prime}(t) \quad \text { for } t \in\right] c, b[
\end{aligned}
$$

respectively. Now using (3.26) in (3.29) and (3.30), we get

$$
\begin{aligned}
& \left.\left.\gamma^{\prime \prime \prime}(t)=-\left(\frac{\nu_{1}}{t-a}+\frac{\lambda_{11}}{(t-a)^{\nu_{1}}}\right) \gamma^{\prime \prime}(t)-\frac{\lambda_{12}}{(t-a)^{2 \nu_{1}}} \gamma^{\prime}(t) \quad \text { for } t \in\right] a, c\right], \\
& \left.\gamma^{\prime \prime \prime}(t)=\left(\frac{\nu_{2}}{b-t}+\frac{\lambda_{21}}{(b-t)^{\nu_{2}}}\right) \gamma^{\prime \prime}(t)-\frac{\lambda_{22}}{(b-t)^{2 \nu_{2}}} \gamma^{\prime}(t) \quad \text { for } t \in\right] c, b[
\end{aligned}
$$

Note that, on account of (3.27) and (3.28), we have $\gamma^{\prime \prime \prime}(t) \leq 0$ for $\left.t \in\right] a, b[$ and, consequently, $\gamma^{\prime \prime}$ is a nonincreasing function. Therefore,

$$
\begin{gathered}
\gamma(t)=\int_{a}^{t} \gamma^{\prime}(s) \mathrm{d} s=(t-a) \gamma^{\prime}(t)-\int_{a}^{t}(s-a) \gamma^{\prime \prime}(s) \mathrm{d} s \\
\left.\leq(t-a) \gamma^{\prime}(t)-\frac{(t-a)^{2}}{2} \gamma^{\prime \prime}(t) \quad \text { for } t \in\right] a, b[
\end{gathered}
$$

and thus (3.31), or (3.32), results in

$$
\begin{aligned}
& \gamma^{\prime \prime \prime}(t) \leq-\left(\frac{\nu_{1}}{t-a}+\frac{\lambda_{11}}{(t-a)^{\nu_{1}}}+p(t) \frac{(t-a)^{2}}{2}\right) \gamma^{\prime \prime}(t) \\
& \left.\left.-\left(\frac{\lambda_{12}}{(t-a)^{2 \nu_{1}}}-p(t)(t-a)\right) \gamma^{\prime}(t)-p(t) \gamma(t) \quad \text { for a.e. } t \in\right] a, c\right],
\end{aligned}
$$

or

$$
\begin{aligned}
& \gamma^{\prime \prime \prime}(t) \leq\left(\frac{\nu_{2}}{b-t}+\frac{\lambda_{21}}{(b-t)^{\nu_{2}}}-p(t) \frac{(t-a)^{2}}{2}\right) \gamma^{\prime \prime}(t) \\
& \left.-\left(\frac{\lambda_{22}}{(b-t)^{2 \nu_{2}}}-p(t)(t-a)\right) \gamma^{\prime}(t)-p(t) \gamma(t) \quad \text { for a.e. } t \in\right] c, b[,
\end{aligned}
$$

respectively. In view of $(2.6)-(2.9),(3.27)$, and (3.28), the latter two inequalities yield

$$
\begin{array}{r}
\gamma^{\prime \prime \prime}(t) \leq-p(t) \sigma(t) \frac{(\tau(t)-t)^{2}}{2} \gamma^{\prime \prime}(t)-p(t) \sigma(t)(\tau(t)-t) \gamma^{\prime}(t)-p(t) \gamma(t) \\
\text { for a.e. } t \in] a, b[.
\end{array}
$$

On the other hand, in view of (3.27),

$$
\int_{t}^{\tau(t)} \gamma^{\prime}(s) \mathrm{d} s \leq 0 \quad \text { if } \tau(t) \leq t
$$


and

$$
\begin{gathered}
\int_{t}^{\tau(t)} \gamma^{\prime}(s) \mathrm{d} s=(\tau(t)-t) \gamma^{\prime}(t)+\int_{t}^{\tau(t)}(\tau(t)-s) \gamma^{\prime \prime}(s) \mathrm{d} s \\
\leq(\tau(t)-t) \gamma^{\prime}(t)+\frac{(\tau(t)-t)^{2}}{2} \gamma^{\prime \prime}(t) \quad \text { if } \tau(t)>t .
\end{gathered}
$$

Thus, from (3.34) and (3.35), we have

$$
\int_{t}^{\tau(t)} \gamma^{\prime}(s) \mathrm{d} s \leq \sigma(t)(\tau(t)-t) \gamma^{\prime}(t)+\sigma(t) \frac{(\tau(t)-t)^{2}}{2} \gamma^{\prime \prime}(t) \quad \text { for a.e. } t \in[a, b] .
$$

Now using (3.36) in (3.33), we obtain

$$
\gamma^{\prime \prime \prime}(t) \leq-p(t) \int_{t}^{\tau(t)} \gamma^{\prime}(s) \mathrm{d} s-p(t) \gamma(t)=-p(t) \gamma(\tau(t)) \quad \text { for a.e. } t \in[a, b] .
$$

Consequently, (3.22)-(3.25), and (3.37) imply that all the assumptions of $[1$, Theorem 2.1] are fulfilled.

Proof of Theorem 2.5. Put

$$
\begin{aligned}
& \beta(t)=1-\frac{1}{(b-a)^{2}}\left((b-t)(t-a) \int_{a}^{t}(b-s)(s-a) g(s) \mathrm{d} s+(t-a)^{2}\right. \\
& \left.-\frac{(b-t)^{2}}{2} \int_{a}^{t}(s-a)^{2} g(s) \mathrm{d} s+\frac{(t-a)^{2}}{2} \int_{t}^{b}(b-s)^{2} g(s) \mathrm{d} s\right) \quad \text { for } t \in[a, b] .
\end{aligned}
$$

We will show that the assumptions of $[1$, Theorem 2.4] are fulfilled. Obviously, $\beta \in \widetilde{C}_{0}(] a, b[; \mathbb{R})$,

$$
\beta(a)=1, \quad \beta^{\prime}(a)=0, \quad \beta(b)=0,
$$

and it can be easily verified that

$$
\begin{aligned}
& \beta^{\prime}(b)=\frac{1}{b-a}\left(\int_{a}^{b}(b-s)(s-a) g(s) \mathrm{d} s-2\right), \\
& \beta^{\prime \prime \prime}(t)=g(t) \quad \text { for a.e. } t \in[a, b] .
\end{aligned}
$$

From (3.38)-(3.40), in view of (2.10), it follows that

$$
\beta^{\prime}(t) \leq 0 \quad \text { for } t \in[a, b] .
$$

Further, put

$$
\gamma(t)=\beta(a+b-t) \quad \text { for } t \in[a, b] .
$$

Then, on account of (3.38), (3.40), and (3.41), we have

$$
\begin{aligned}
& \gamma^{\prime \prime \prime}(t)=-g(t) \quad \text { for a.e. } t \in[a, b], \\
& \gamma(a)=0, \quad \gamma^{\prime}(a) \geq 0, \quad \gamma(b)=1,
\end{aligned}
$$


whence, according to [1, Remark 2.3, Theorem 2.10], it follows that

$$
\gamma(t)>0 \quad \text { for } t \in] a, b[\text {. }
$$

However, the latter inequality together with (3.38) and (3.42) results in

$$
\beta(t)>0 \quad \text { for } t \in[a, b[\text {. }
$$

Finally, in view of (3.38) and (3.41), we have

$$
\beta(\mu(t)) \leq 1 \quad \text { for a.e. } t \in[a, b],
$$

which, together with (3.40), results in

$$
\beta^{\prime \prime \prime}(t) \geq g(t) \beta(\mu(t)) \quad \text { for a.e. } t \in[a, b] .
$$

Consequently, (3.41), (3.43), and (3.45) imply that all the assumptions of [1, Theorem 2.4] are fulfilled.

Proof of Corollary 2.6. It immediately follows from Theorem 2.5 because

$$
\int_{a}^{b}(b-s)(s-a) g(s) \mathrm{d} s \leq \frac{(b-a)^{2}}{4} \int_{a}^{b} g(s) \mathrm{d} s .
$$

Proof of Theorem 2.7. Put

$$
\beta(t)=(b-t)^{1+\frac{\sqrt{3}}{3}}(t-a+\omega)^{1-\frac{\sqrt{3}}{3}} \quad \text { for } t \in[a, b] .
$$

Then, obviously, $\beta \in \widetilde{C}_{0}(] a, b[; \mathbb{R}),(3.43)$ holds,

$$
\beta^{\prime}(a)=0, \quad \beta^{\prime}(b)=0,
$$

and

$$
\left.\beta^{\prime \prime \prime}(t)=\frac{2 \sqrt{3}(b-a+\omega)^{3}}{9(b-t)^{3}(t-a+\omega)^{3}}(b-t)^{1+\frac{\sqrt{3}}{3}}(t-a+\omega)^{1-\frac{\sqrt{3}}{3}} \quad \text { for } t \in\right] a, b[.
$$

From (3.47) and (3.48), it follows that (3.41) holds. Moreover, using (2.11) in (3.48), on account of (3.46), we get (3.45). Thus, all the assumptions of [1, Theorem 2.4] are fulfilled.

Proof of Theorem 2.8. Assume $c \in] a, b[$; the cases $c=a$ and $c=b$ can be proved analogously. Without loss of generality we can assume that (2.4) and (2.5) are fulfilled as equalities. Define functions $\rho_{i}(i=1,2)$ by $(3.16)$ and (3.17), respectively. Then (3.18)-(3.21) hold. Define $z$ by (3.22) and put

$$
\beta(t)=\int_{t}^{b} z(s) \mathrm{d} s \quad \text { for } t \in[a, b] .
$$

We will show that $\beta$ satisfies the assumptions of [1, Theorem 2.4]. Obviously, $\beta \in \widetilde{C}_{0}(] a, b[; \mathbb{R})$ and

$$
\beta(b)=0, \quad \beta(t)>0 \quad \text { for } t \in[a, b[.
$$

Moreover, in view of (3.22), we have

$$
\left.\beta^{\prime}(t)<0 \quad \text { for } t \in\right] a, b[.
$$


Furthermore, (3.22) and (3.49) yield

$$
\beta^{\prime \prime}(t)= \begin{cases}(t-a)^{-\nu_{1}} \rho_{1}(t) \beta^{\prime}(t) & \text { for } t \in] a, c], \\ -(b-t)^{-\nu_{2}} \rho_{2}(t) \beta^{\prime}(t) & \text { for } t \in] c, b[.\end{cases}
$$

In view of (3.18) and (3.51), we have

$$
\left.\beta^{\prime \prime}(t)<0 \quad \text { for } t \in\right] a, c\left[, \quad \beta^{\prime \prime}(t)>0 \quad \text { for } t \in\right] c, b[.
$$

Finally, with respect to (3.20), or (3.21), from (3.52) we obtain

$$
\begin{aligned}
& \beta^{\prime \prime \prime}(t)=-\nu_{1}(t-a)^{-\nu_{1}-1} \rho_{1}(t) \beta^{\prime}(t)-(t-a)^{-2 \nu_{1}} \lambda_{11} \rho_{1}(t) \beta^{\prime}(t) \\
& \left.\left.-(t-a)^{-2 \nu_{1}} \lambda_{12} \beta^{\prime}(t) \quad \text { for } t \in\right] a, c\right],
\end{aligned}
$$

or

$$
\begin{aligned}
& \beta^{\prime \prime \prime}(t)=-\nu_{2}(b-t)^{-\nu_{2}-1} \rho_{2}(t) \beta^{\prime}(t)-(b-t)^{-2 \nu_{2}} \lambda_{21} \rho_{2}(t) \beta^{\prime}(t) \\
& \left.-(b-t)^{-2 \nu_{2}} \lambda_{22} \beta^{\prime}(t) \quad \text { for } t \in\right] c, b[,
\end{aligned}
$$

respectively. Now using (3.52) in (3.54) and (3.55), we get

$$
\begin{aligned}
& \left.\left.\beta^{\prime \prime \prime}(t)=-\left(\frac{\nu_{1}}{t-a}+\frac{\lambda_{11}}{(t-a)^{\nu_{1}}}\right) \beta^{\prime \prime}(t)-\frac{\lambda_{12}}{(t-a)^{2 \nu_{1}}} \beta^{\prime}(t) \quad \text { for } t \in\right] a, c\right], \\
& \left.\beta^{\prime \prime \prime}(t)=\left(\frac{\nu_{2}}{b-t}+\frac{\lambda_{21}}{(b-t)^{\nu_{2}}}\right) \beta^{\prime \prime}(t)-\frac{\lambda_{22}}{(b-t)^{2 \nu_{2}}} \beta^{\prime}(t) \quad \text { for } t \in\right] c, b[.
\end{aligned}
$$

Note that, on account of (3.51) and (3.53), we have $\beta^{\prime \prime \prime}(t) \geq 0$ for $\left.t \in\right] a, b[$ and, consequently, $\beta^{\prime \prime}$ is a nondecreasing function. Therefore,

$$
\begin{aligned}
& \beta(t)=-\int_{t}^{b} \beta^{\prime}(s) \mathrm{d} s=-(b-t) \beta^{\prime}(t)-\int_{t}^{b}(b-s) \beta^{\prime \prime}(s) \mathrm{d} s \\
& \left.\leq-(b-t) \beta^{\prime}(t)-\frac{(b-t)^{2}}{2} \beta^{\prime \prime}(t) \quad \text { for } t \in\right] a, b[,
\end{aligned}
$$

and thus, (3.56) or (3.57) results in

$$
\begin{aligned}
& \beta^{\prime \prime \prime}(t) \geq-\left(\frac{\nu_{1}}{t-a}+\frac{\lambda_{11}}{(t-a)^{\nu_{1}}}-g(t) \frac{(b-t)^{2}}{2}\right) \beta^{\prime \prime}(t) \\
& \left.\left.-\left(\frac{\lambda_{12}}{(t-a)^{2 \nu_{1}}}-g(t)(b-t)\right) \beta^{\prime}(t)+g(t) \beta(t) \quad \text { for a.e. } t \in\right] a, c\right],
\end{aligned}
$$

or

$$
\begin{gathered}
\beta^{\prime \prime \prime}(t) \geq\left(\frac{\nu_{2}}{b-t}+\frac{\lambda_{21}}{(b-t)^{\nu_{2}}}+g(t) \frac{(b-t)^{2}}{2}\right) \beta^{\prime \prime}(t) \\
\left.-\left(\frac{\lambda_{22}}{(b-t)^{2 \nu_{2}}}-g(t)(b-t)\right) \beta^{\prime}(t)+g(t) \beta(t) \quad \text { for a.e. } t \in\right] c, b[
\end{gathered}
$$

respectively. In view of (2.12)-(2.15), (3.51), and (3.53), the latter two inequalities yield

$$
\beta^{\prime \prime \prime}(t) \geq g(t) \frac{(t-\mu(t))^{2}}{2} \beta^{\prime \prime}(t)-g(t)(t-\mu(t)) \beta^{\prime}(t)+g(t) \beta(t)
$$


On the other hand,

$$
\begin{gathered}
\int_{\mu(t)}^{t} \beta^{\prime}(s) \mathrm{d} s=(t-\mu(t)) \beta^{\prime}(t)-\int_{\mu(t)}^{t}(s-\mu(t)) \beta^{\prime \prime}(s) \mathrm{d} s \\
\geq(t-\mu(t)) \beta^{\prime}(t)-\frac{(t-\mu(t))^{2}}{2} \beta^{\prime \prime}(t) \quad \text { for a.e. } t \in[a, b] .
\end{gathered}
$$

Now using (3.59) in (3.58), we obtain

$$
\beta^{\prime \prime \prime}(t) \geq-g(t) \int_{\mu(t)}^{t} \beta^{\prime}(s) \mathrm{d} s+g(t) \beta(t)=g(t) \beta(\mu(t)) \quad \text { for a.e. } t \in[a, b] .
$$

Consequently, (3.50), (3.51), and (3.60) imply that all the assumptions of [1, Theorem 2.4] are fulfilled.

Proof of Theorem 2.9. If $g \equiv 0$, then the conclusion of theorem follows from [1, Remarks 1.4 and 2.3]. Therefore, we assume that

$$
\int_{a}^{b} g(s) \mathrm{d} s>0
$$

Put

$$
\begin{aligned}
& \beta(t)=\frac{1}{(b-a)^{2}}\left((b-t)(t-a) \int_{t}^{b}(b-s)(s-a) g(s) \mathrm{d} s\right. \\
& \left.-\frac{(t-a)^{2}}{2} \int_{t}^{b}(b-s)^{2} g(s) \mathrm{d} s+\frac{(b-t)^{2}}{2} \int_{a}^{t}(s-a)^{2} g(s) \mathrm{d} s\right) \quad \text { for } t \in[a, b] .
\end{aligned}
$$

We will show that $\beta$ satisfies the assumptions of [1, Theorem 2.5] with $\ell$ defined by (1.4). It can be easily verified that

$$
\begin{aligned}
& \beta^{\prime \prime \prime}(t)=g(t) \quad \text { for a.e. } t \in[a, b], \\
& \beta(a)=0, \quad \beta^{\prime}(b)=0, \quad \beta(b)=0 .
\end{aligned}
$$

Defining

$$
\gamma(t)=\beta(a+b-t) \quad \text { for } t \in[a, b],
$$

from (3.63) and (3.64) we obtain

$$
\begin{aligned}
& \gamma^{\prime \prime \prime}(t)=-g(a+b-t) \quad \text { for a.e. } t \in[a, b], \\
& \gamma(a)=0, \quad \gamma^{\prime}(a)=0, \quad \gamma(b)=0 .
\end{aligned}
$$

Therefore, according to [1, Remark 2.3, Theorem 2.10] and the inequality (3.61), we have $\gamma(t)>0$ for $t \in] a, b[$ and, consequently,

$$
\beta(t)>0 \quad \text { for } t \in] a, b[\text {. }
$$

Furthermore, (2.16) and (3.62) imply (3.44), which, when used in (3.63), yields (3.45). Finally, $\beta \in \widetilde{C}_{0}(] a, b[; \mathbb{R})$ and (3.45), (3.64), and (3.65) imply that all the assumptions of $[1$, Theorem 2.5] are fulfilled. 
Proof of Corollary 2.10. Define $\beta$ by (3.62) and put

$$
\gamma(t)=\beta(a+b-t) \quad \text { for } t \in[a, b] .
$$

Then $\gamma$ satisfies (3.2) with

$$
p(t)=g(a+b-t) \quad \text { for a.e. } t \in[a, b] .
$$

Analogously to the proof of Corollary 2.2, in view of (2.17) and (3.67), it can be easily verified that (3.14) holds where $\left.t_{0} \in\right] a, b[$ is such that (3.9) is satisfied. Thus, in view of (3.66), we have (3.44) and, consequently, (2.16) is fulfilled.

Proof of Theorem 2.11. Put

$$
\beta(t)=(b-t)^{1+\frac{\sqrt{3}}{3}}(t-a)^{1-\frac{\sqrt{3}}{3}} \quad \text { for } t \in[a, b] .
$$

Obviously, $\beta \in \widetilde{C}_{0}(] a, b[; \mathbb{R}), \beta(t)>0$ for $\left.t \in\right] a, b[$,

$$
\beta(a)=0, \quad \beta^{\prime}(b)=0, \quad \beta(b)=0,
$$

and

$$
\beta^{\prime \prime \prime}(t)=\frac{2 \sqrt{3}(b-a)^{3}}{9(b-t)^{3}(t-a)^{3}}(b-t)^{1+\frac{\sqrt{3}}{3}}(t-a)^{1-\frac{\sqrt{3}}{3}} \quad \text { for a.e. } t \in[a, b] .
$$

Using (2.18) in the latter equality, in view of (3.68), we get

$$
\beta^{\prime \prime \prime}(t) \geq g(t) \beta(\mu(t)) \quad \text { for a.e. } t \in[a, b] .
$$

Thus, all the assumptions of [1, Theorem 2.5] are fulfilled.

\section{REFERENCES}

[1] R. Hakl, On a two-point boundary value problem for third-order linear functional differential equations. Part I, Math. Appl. 1 (2012), 57-78.

Robert Hakl, Institute of Mathematics, Academy of Sciences of the Czech Republic, Žižkova 22, 61662 Brno, Czech Republic

e-mail: hakl@ipm.cz 
